\title{
Scholte-Stoneley waves on an immersed solid dihedral: Generation, propagation and scattering effects
}

\author{
Ebrahim Lamkanfi ${ }^{a, *}$, Nico F. Declercq ${ }^{b}$, Wim Van Paepegem ${ }^{a}$, Joris Degrieck ${ }^{a}$ \\ ${ }^{a}$ Ghent University, Department of Materials Science and Engineering, Technologiepark-Zwijnaarde 903, 9052 Zwijnaarde, Belgium

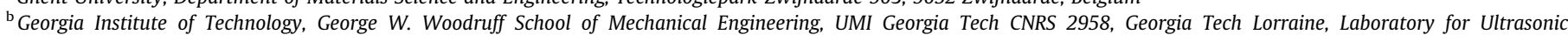 \\ Nondestructive Evaluation, 2 rue Marconi, 57070 Metz, France
}

\section{A R T I C L E I N F O}

\section{Article history:}

Received 30 December 2013

Received in revised form 19 February 2014

Accepted 20 February 2014

Available online $\mathrm{xxxx}$

\section{Keywords:}

Scholte-Stoneley waves

Finite element method

Diffraction

Dihedral

\begin{abstract}
A B S T R A C T
Scholte-Stoneley wave propagation on a dihedral and more precisely the diffraction effects occurring at the corners, has since long been of high importance for nondestructive testing of materials and structures. Experimental investigations have been reported in the past. Simulations based on radiation mode theory have been published before, explaining the only situation for which the model is applicable namely rectangular corners. The current report describes an investigation applying finite element simulations. Results are obtained not only for rectangular corners but for any possible corner angle. The outcome is in agreement with reported experiments. Moreover a critical corner angle is found below and beyond which different diffraction phenomena occur. The study is performed for different isotropic solids.
\end{abstract}

() 2014 Published by Elsevier B.V.

\section{Introduction}

Surface acoustic waves are important for nondestructive testing of materials. Their propagation properties are well-known, but scattering effects when they encounter obstacles, such as an edge, still require investigations. A well-known type of surface acoustic waves is the leaky Rayleigh wave. Gipson and Marston [1] for instance have reported investigations of scattering and backscattering of Rayleigh waves at (rectangular) edges of cubes. Comparable to leaky Rayleigh waves, Scholte-Stoneley waves [2,3] are essentially surface waves propagating on the interface between an isotropic solid and a liquid. Contrary to leaky Rayleigh waves, their energy is situated mostly on the liquid side and less on the solid side. The velocity of Scholte-Stoneley waves is smaller but still close to the acoustic bulk wave velocity in water. Different techniques have been developed in the past to generate such waves on an interface. They are more difficult to generate because Snell's law, in combination with the fact that their velocity is lower than the one in water, determines that the angle of incidence must be complex, i.e. 90 degrees plus an imaginary number. In other words if impinging sound is used, then the sound must be incident at grazing angle and must have the shape of an inhomogeneous wave [4]. That's a practical limitation overcome by more sophisticated generation techniques [5-8].

\footnotetext{
* Corresponding author. Tel.: +32 93310434.

E-mail address: ebrahim.lamkanfi@ugent.be (E. Lamkanfi).
}

Tinel and Duclos [9] used a special type of transducer, a so-called interdigital transducer [10], which allows the generation of surface waves at the surface of piezoelectric crystals. It is also shown [11] that this type of transducer is able to generate Scholte-Stoneley waves when the crystal is immersed in a liquid. This is depicted in Fig. 1 where the transducer is in contact with a solid dihedral that is completely immersed in water. The experimental setup of Tinel and Duclos enables an investigation of the influence of the edge angle on the diffracted sound patterns caused by an incident Scholte-Stoneley wave. A sophisticated measuring system, applying a rotating measuring device, permitted quantification of the sound pressure around the corner.

The current paper presents a numerical study similar to the experimental investigations done by Tinel and Duclos. As in the paper by Tinel and Duclos, we take into account the following sign convention: the $\theta$-angle is positive in the upper part of Fig. 1 and negative elsewhere. At specific angles, shown as arrows in Fig. 1, Duclos and Tinel observe different scattered waves [9]. First of all, the highest amplitudes are found in-line with the solid-liquid interface, i.e. at an $\theta$-angle of $0^{\circ}$. This forward diffracted sound field is very characteristic for the diffraction of Scholte-Stoneley waves at the extremity of the solid plate. It is found in other reports that a similar effect is not observed for leaky Rayleigh waves [12]. In addition to the forward diffracted sound field, smaller but still significant amplitudes are measured at other $\theta$-angles: first a reflected Rayleigh angle at the critical Rayleigh angle $\theta_{R}$ as seen in Fig. 1 ; second, a transmitted Scholte-Stoneley wave at $-180^{\circ}+\gamma$; third, a 


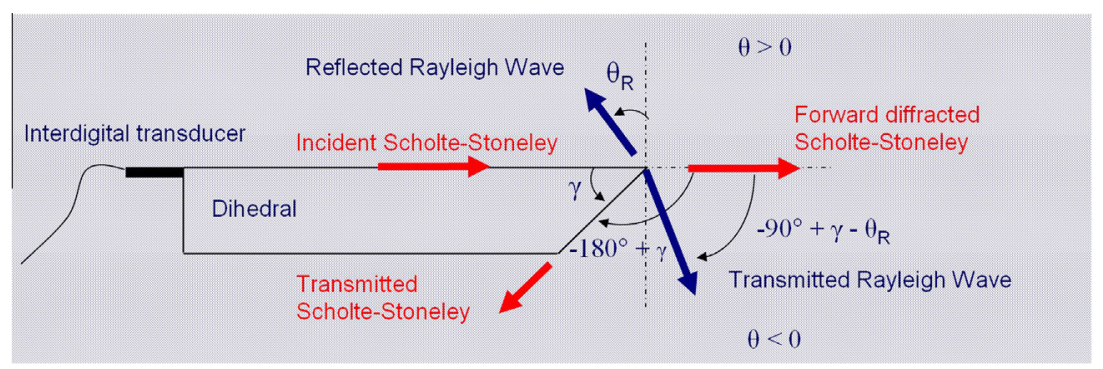

Fig. 1. Diffraction of an incident Scholte-Stoneley wave at the corner of a solid dihedral.

transmitted Rayleigh wave at $-90^{\circ}+\gamma-\theta_{R}$. The experiments done by Tinel and Duclos [9] consist of measurements with dihedral corner angles ranging from $45^{\circ}$ to $90^{\circ}$. For all these dihedral angles, similar diffraction phenomena are observed.

Earlier a theoretical study carried out by Briers et al. [13], confirmed some of the experimental results found by Tinel and Duclos [9]. The study was based on the radiation mode theory [14] that consists of the construction of a set of acoustic modes (radiation modes and eigenmodes) of the solid/liquid system under consideration. Despite of the satisfactory results obtained with this approach, the method remains very cumbersome [13] and is only applicable to solid corners of $90^{\circ}$. The current report presents numerical simulations based on different approach, namely the finite element technique, for the varying dihedral corner angles experimentally investigated by Tinel and Duclos [9]. First, a detailed description of phenomena appearing at $90^{\circ}$ is given, then the situation for dihedral angles different from $90^{\circ}$ is tackled. Furthermore, a 'critical angle' will be defined of the dihedral corner constituting a sudden diffraction pattern switch.

\section{Formulation of boundary conditions in terms of analytical solutions}

In order to study Scholte-Stoneley waves in a finite element approach it is first necessary to obtain their displacement field analytically. This field is consequently incorporated as a boundary condition in the finite element model. To do so the three continuity equations for normal displacement Eq. (1) and normal stresses Eq. (2) and the vanishing tangential stresses Eq. (3) along the solid-liquid interface $z=0$ must be fulfilled (Fig. 2):

$$
\begin{aligned}
& u_{S, z}=u_{L, z} \\
& T_{S, z z}=T_{L, z z} \\
& T_{L, x z}=0
\end{aligned}
$$

The quantities in Eqs. (1)-(3) are described in terms of the potential functions $\Phi_{S}$ and $\vec{\Psi}_{S}$ in the solid, the potential function $\Phi_{L}$ in the liquid and the displacement functions $\vec{u}_{S}$ and $\vec{u}_{L}$ as

$$
\begin{aligned}
& \Phi_{S}=A \cdot e^{k_{k_{z} z}} \cdot e^{i\left(k_{R} x-\omega t\right)} \\
& \vec{\Psi}_{S}=B \cdot e^{k_{S z} z} \cdot e^{i\left(k_{R} x-\omega t\right)} \\
& \Phi_{L}=C \cdot e^{-k_{L z} x} \cdot e^{i\left(k_{R} z-\omega t\right)} \\
& \vec{u}_{S}=\vec{\nabla} \Phi_{S}+\vec{\nabla} \times \vec{\Psi}_{S} \\
& \vec{u}_{L}=\vec{\nabla} \Phi_{L}
\end{aligned}
$$

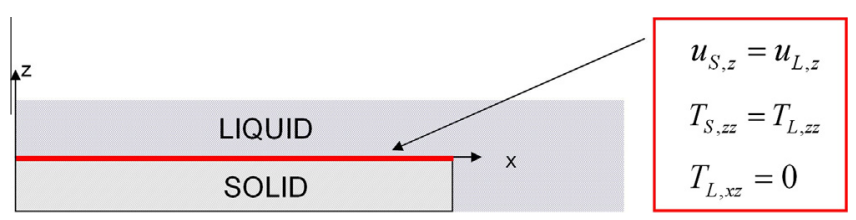

Fig. 2. Mechanical continuity equations at the solid-liquid interface applied to calculate analytically Scholte-Stoneley wave properties. whereby the wave vector components satisfy the dispersion relations

$k_{x}^{2}+k_{l z}^{2}=k_{l}^{2}=\left(\frac{\omega}{v_{l}}\right)^{2}$

$k_{x}^{2}+k_{s z}^{2}=k_{s}^{2}=\left(\frac{\omega}{v_{s}}\right)^{2}$

$k_{x}^{2}+k_{L z}^{2}=k_{L}^{2}=\left(\frac{\omega}{v_{L}}\right)^{2}$

where the solid (S) is characterized by its density $\rho_{S}$, its longitudinal sound velocity $v_{l}$ and its transversal sound velocity $v_{s}$, the liquid (L) by its density $\rho_{L}$ and its longitudinal sound velocity $v_{L}$ and where $\omega$ is the circular frequency, $k_{l z}$ the $z$-component of the longitudinal wave vector in the solid, $k_{s z}$ the $z$-component of the shear wave vector in the solid and $k_{L z}$ the $z$-component of the longitudinal wave vector in the liquid. Moreover, the three amplitudes $A, B, C$ are arbitrarily chosen. Substitution of Eqs. (4)-(6) into Eqs. (7) and (8) and use of the Cauchy strain formulation

$\epsilon_{m, i j}=\frac{1}{2}\left(\frac{\partial u_{m, i}}{\partial x_{j}}+\frac{\partial u_{m, j}}{\partial x_{i}}\right)$

with Hooke's law Eq. (13),

$T_{m, i j}=\sum \lambda \epsilon_{m, k k} \delta_{i j}+2 \mu \epsilon_{m, i j}$

where $i, j=\{1,2\}, m=\{S, L\}, x_{1}=x, x_{2}=z, \delta_{i j}$ the Kronecker symbol, $\lambda$ and $\mu$ the Lamé constants, leads to the characteristic equation for surface waves along the solid-liquid interface

$4 k_{R}^{2} k_{s z} k_{l z}-\left(k_{R}^{2}+k_{s z}^{2}\right)=i \frac{\rho_{L}}{\rho_{S}} \frac{k_{l z} k_{s z}^{4}}{\sqrt{k_{L z}^{2}-k_{R}^{2}}}$

with $k_{R}=\frac{\omega}{v_{R}}$ the unknown wave number for the Scholte-Stoneley waves. Eq. (14) is solved for $k_{R}$, applying the dispersion equations Eqs. (9)-(11) and the following material parameters for an aluminum-water system with $\rho_{L}=1000 \mathrm{~kg} / \mathrm{m}^{3}, \rho_{S}=2700 \mathrm{~kg} / \mathrm{m}^{3}$, $v_{l}=6420 \mathrm{~m} / \mathrm{s}, v_{\mathrm{s}}=3040 \mathrm{~m} / \mathrm{s}$ and $v_{L}=1480 \mathrm{~m} / \mathrm{s}$. A $5 \mathrm{MHz}$ Scholte-Stoneley wave results in a wave velocity of $1476.47 \mathrm{~m} / \mathrm{s}$ and a wavelength of $0.2953 \mathrm{~mm}$. The penetration depth turns out to be less than 3 wavelengths in the solid and less than 11 wavelengths in the liquid. This can be observed in Fig. 3a and b where the decay of the displacement of the Scholte-Stoneley away from the liquidsolid interface is respectively given in the liquid $(z>0)$ and the solid $(z<0)$.

\section{Finite element model}

When analytical methods are cumbersome or impossible to apply, the use of numerical methods such as the finite element method becomes apparent, not only in acoustics. The solution offered by this numerical method in general approximates scientific or engineering experiments very well, because it represents a general class of techniques to solve partial differential equations. The 


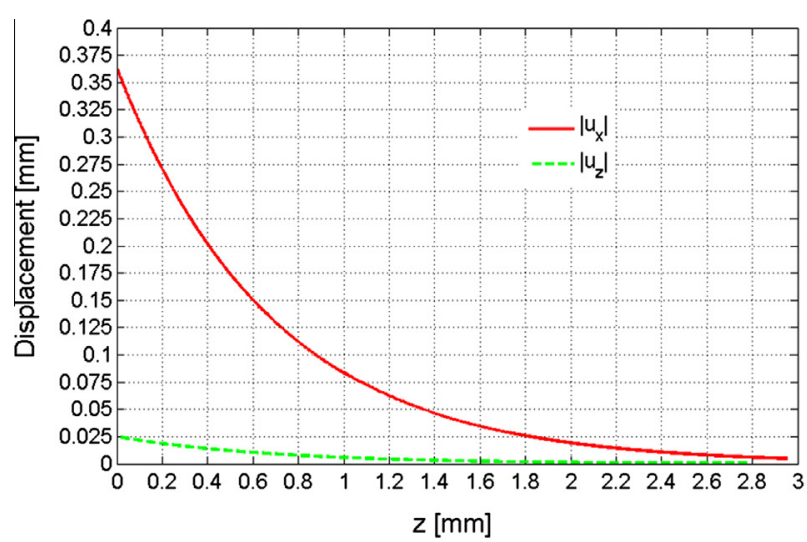

(a)

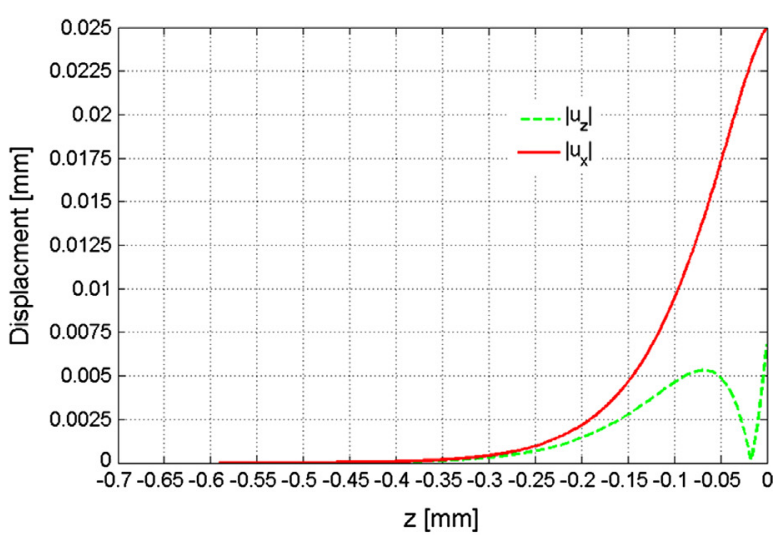

(b)

Fig. 3. Displacement curves in the liquid $(z>0)$ (a) and the solid $(z<0)$ (b).

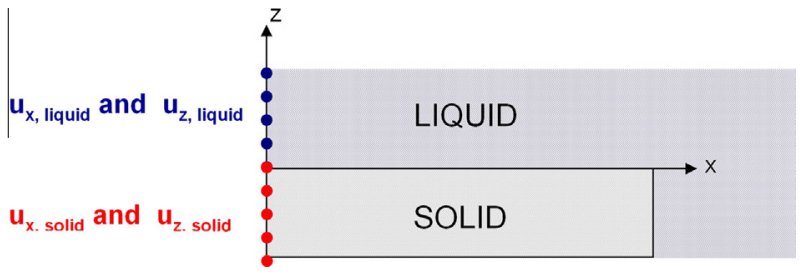

Fig. 4. Boundary conditions are applied to mimic a Scholte-Stoneley wave generating sound source.

coupled acoustic-structural problem discussed above is treated by dividing the geometry in a number of elements and solving the governing equations in those elements iteratively. Consequently, the finite element method enables the investigation of scattering and diffraction phenomena taking place when Scholte-Stoneley waves reach the dihedral edge. It is important to mention that in order to capture the sound field correctly at a scale smaller than one wavelength, the number of elements should be accordingly high.
Unlike the experiments, achieved using an interdigital transducer, the numerical simulations apply appropriate boundary conditions to force Scholte-Stoneley wave generation, based on the results obtained above. This procedure is represented by Fig. 4, where the displacement fields $u_{x}$ and $u_{z}$ are applied at $x=0$ for the solid boundary, whereas for the liquid region the displacements are transformed as a pressure boundary by means of the expression

$p=-B_{f} \cdot\left(\frac{\partial u_{L, x}}{\partial x}+\frac{\partial u_{L, z}}{\partial z}\right)$

in which $B_{f}$ represents the bulk modulus of the fluid.

\subsection{Simulation of Scholte-Stoneley waves}

Prior to proceeding towards scattering effects, it is important to investigate the propagation of Scholte-Stoneley waves with the finite element approach and to compare with the analytically obtained Scholte-Stoneley wave. The first problem to resolve is the correct application of the boundary conditions imposed by the analytical Scholte-Stoneley wave distribution ( $z$-axis in

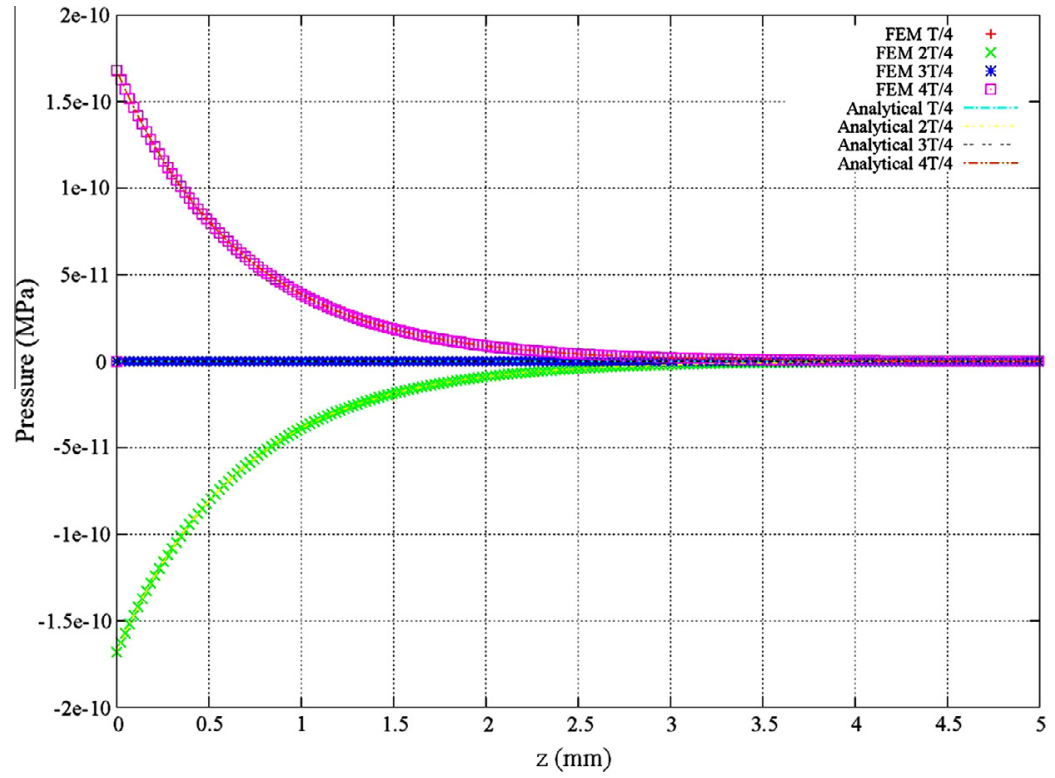

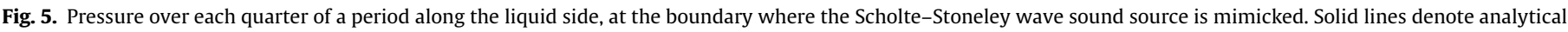
results, whereas the probed values are given by symbolic representations as indicated at the top right corner of the figure. 


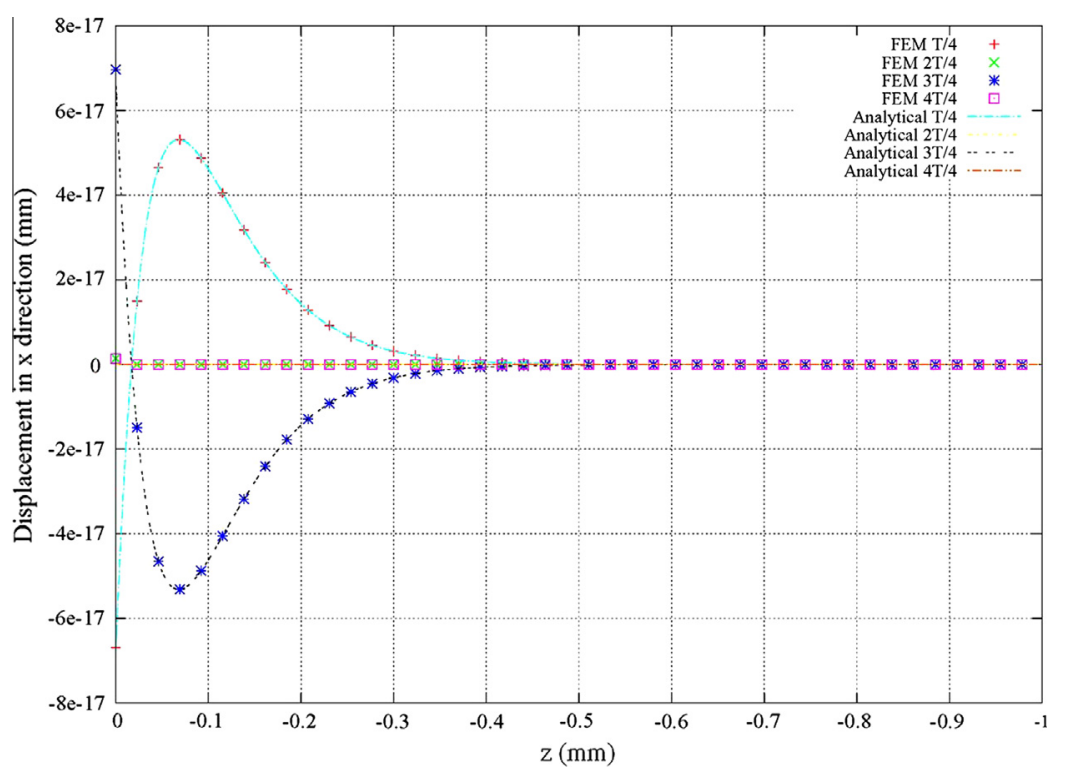

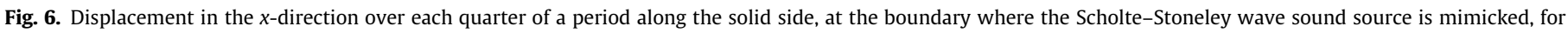
analytically (solid lines) and numerically (probed values) derived Scholte-Stoneley waves.

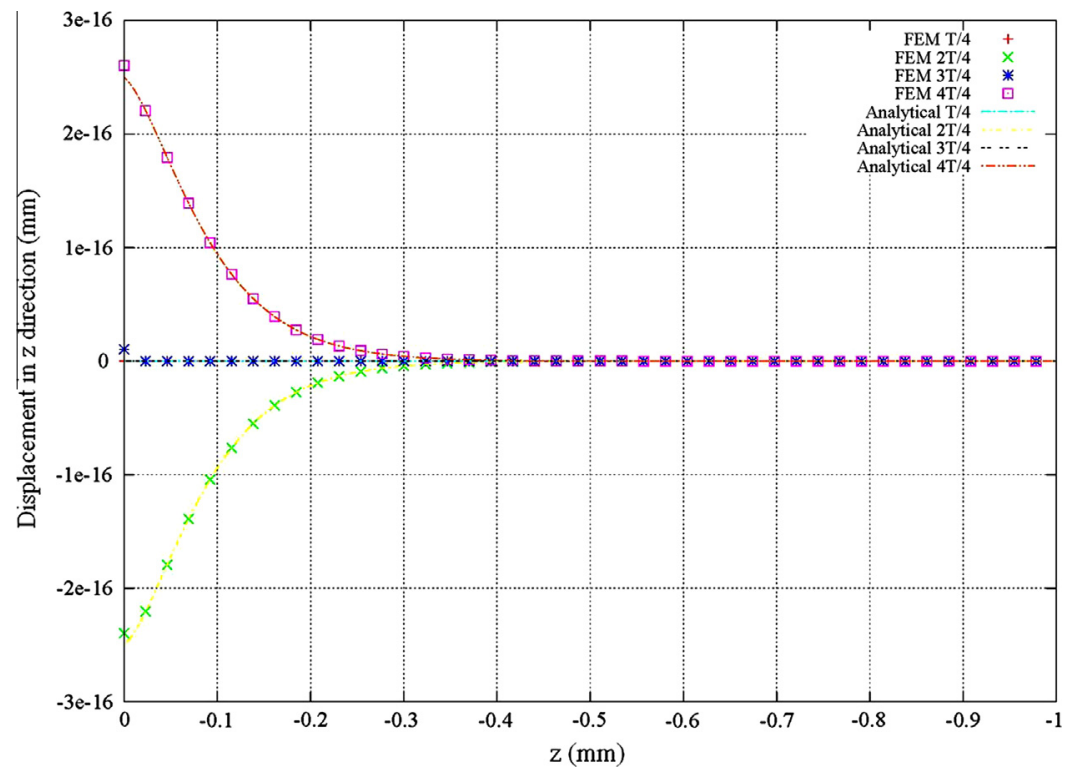

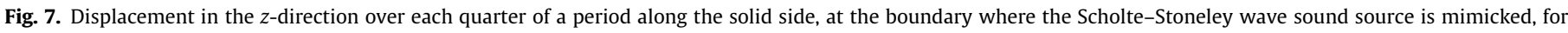
theoretically (solid lines) and numerically (probed values) derived Scholte-Stoneley waves.

Fig. 4). The mesh density in this regard is important because the displacement fields are probed at the nodes of the mesh. For these elements an average length of $2 \mathrm{E}-5 \mathrm{~m}$ is considered so that the sound field can be correctly captured at the boundary of the two media. Comparison with the theoretical curves shows that the obtained displacement curves in the solid and the pressure curve in the liquid match very well. This can be readily observed in Fig. 5 where the pressure distribution along the vertical edge at $x=0$ in the liquid medium $(z>0)$ is shown. The theoretical curves are given in solid lines, whereas the finite element curves extracted along this line are given in discrete points. The necessary match is clearly visible. Also for the displacement fields $u_{1}$ and $u_{2}$, along the solid boundary $(z \leqslant 0)$, the same comparison is made. It is clear that an equally accurate match is obtained as seen in Figs. 6 and 7.

In Fig. 8a the forward propagating Scholte-Stoneley wave, found through application of the aforementioned boundary conditions, is depicted. In the upper parts the pressure distribution in the liquid is shown, whereas in the lower parts the $z$-displacement field of the solid medium is shown. Comparison between the analytical results of Fig. $8 \mathrm{a}$ and $\mathrm{b}$ and the finite element results of Fig. $8 \mathrm{c}$ and d clearly shows that the pressure wave and the vertical displacement, which are in phase, are exactly the same. This match signifies also that the necessary mechanical continuity equations at the interface between the solid and the liquid medium are equally guaranteed by the finite element method as by the analytical method.

\section{Interaction with a $\mathbf{9 0}^{\circ}$ solid edge}

The previous section shows that the implementation of the Scholte-Stoneley waves in the finite element model is correct. 


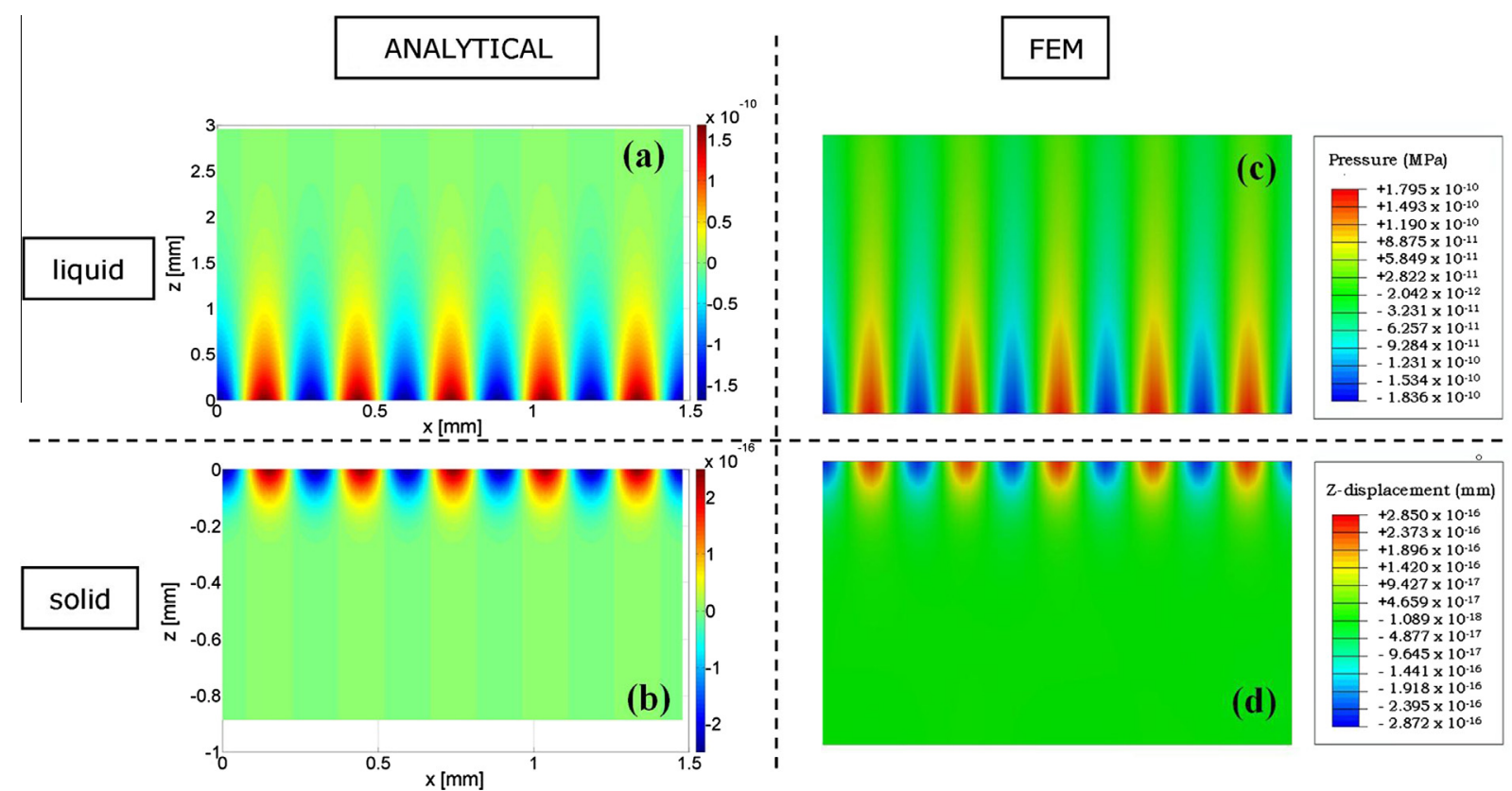

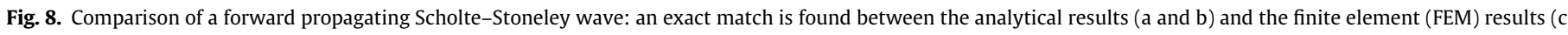
and $\mathrm{d}$ ) in the solid and liquid region.

Here we proceed to the interaction of these surface waves with the edge of an immersed solid plate. The configuration studied in the current and the subsequent sections is a liquid/solid/liquid system, being an $1 \mathrm{~cm}$ thick immersed solid plate. To enable comparison with the experimental results as well as the radiation mode results reported in the literature and described earlier, the aluminum-inwater configuration is used as in Tinel and Duclos [9] and Briers et al. [13]. As mentioned in the introduction, we apply the finite element method to retrieve the diffracted waves discussed in Section 1. For this, a finite element model is developed in which a Scholte-Stoneley wave is released along the liquid-solid interface towards the edge having $\gamma$ equal to $90^{\circ}$. When the numerically modeled Scholte-Stoneley wave propagates towards the edge, a specific diffraction pattern in the surrounding liquid appears (Fig. 9).

Different distinct characteristics can readily be recognized. First, the forward diffracted Scholte-Stoneley wave at $\theta=0^{\circ}$ is clearly noticeable. As reported in cited works $[9,13]$ it is obvious that this forward beam has the highest intensity. Second a reflected Rayleigh re-radiation field is observed at the Rayleigh angle for a water-aluminum interface of $\theta_{R}$ of $31^{\circ}$. Third, a transmitted Scholte-Stoneley wave appears at $-180^{\circ}+90^{\circ}=-90^{\circ}$, and also a transmitted Rayleigh wave at $-90^{\circ}+90^{\circ}-31^{\circ}=-31^{\circ}$. It is therefore shown that the developed finite element model confirms experimental results found by Tinel et al. [9] and theoretical results found by Briers et al. [13]. Not explicitly mentioned in cited works $[9,13]$ is the appearance of a 'trailing field' caused by Rayleigh waves leaking energy back into the liquid. This phenomenon is visible in Fig. 9 for the reflected as well as the transmitted Rayleigh wave. Also the fact that a Rayleigh wave travels around a solid edge when it encounters one [12,15], is clearly found in Fig. 9. The latter is seen not only on the top corner, but even on the bottom corner $90^{\circ}$ of the solid plate. Hence the finite element model produces results in agreement with experiments and consistency with known acoustic scattering effects. The real power of the technique emerges however in cases not theoretically studied before. Dihedral angles $\gamma$ different from $90^{\circ}$ have been experimentally investigated by Tinel and Duclos and are studied numerically in the following section.

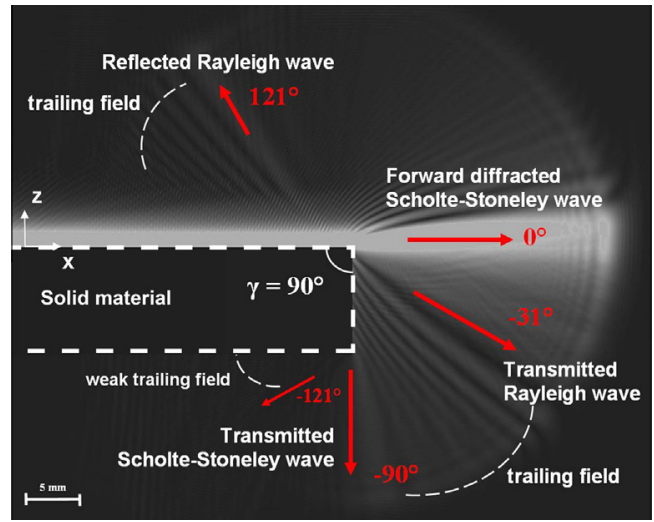

Fig. 9. Diffraction of a forward propagating Scholte-Stoneley wave at the edge of solid plate.

\section{Interaction with a dihedral with different $\gamma$ angles}

Whereas the radiation mode theory is limited to $90^{\circ}$ corner angles, the finite element method equally works with other angles. The aluminum-water system, discussed above, is reconsidered and the angle $\gamma$ of the solid dihedral is changed from $90^{\circ}$ to around $45^{\circ}$. Attention is paid to diffraction waves as in earlier sections, but also to other phenomena. In Fig. 10 the results are shown for six $\gamma$ angles including the $90^{\circ}$ one discussed before, i.e. $\gamma=90^{\circ}, 72^{\circ}, 64^{\circ}, 61^{\circ}, 55^{\circ}, 48^{\circ}$. In addition to the forward propagating Scholte-Stoneley wave always at $\theta=0^{\circ}$, the reflected/transmitted Rayleigh angles and the transmitted Scholte-Stoneley angle are clearly visible at their respective angles of $\theta_{R},-90^{\circ}+\gamma-\theta_{R}$ and $-180^{\circ}+\gamma$. Furthermore with a decreasing $\gamma$ angle, another observation is made. There appears to be a significant null-zone pointing in the direction of $\left(\theta=-31^{\circ}\right)$ in Fig. 10a, incorporated in the trailing field caused by the transmitted Rayleigh wave, that rotates downwards along with the corresponding Rayleigh angle for subsequent decreasing $\gamma$ angles. Furthermore this null field becomes less distinguishable with decreasing $\gamma$ and is not even noticable for $\gamma$ angles smaller than $64^{\circ}$. Simultaneously 

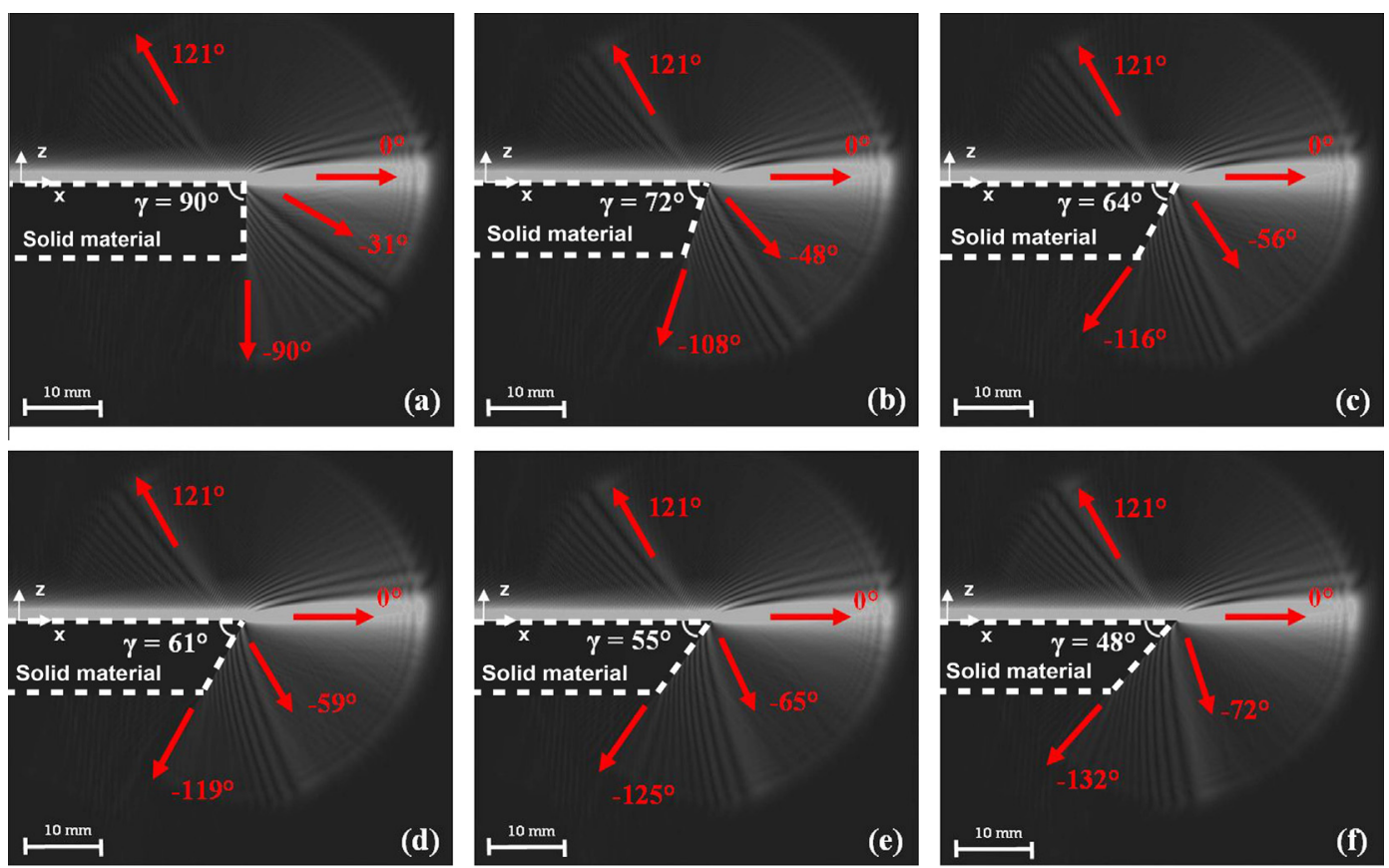

Fig. 10. Diffraction angles for a $5 \mathrm{MHz}$ Scholte-Stoneley wave in a aluminum-water system.
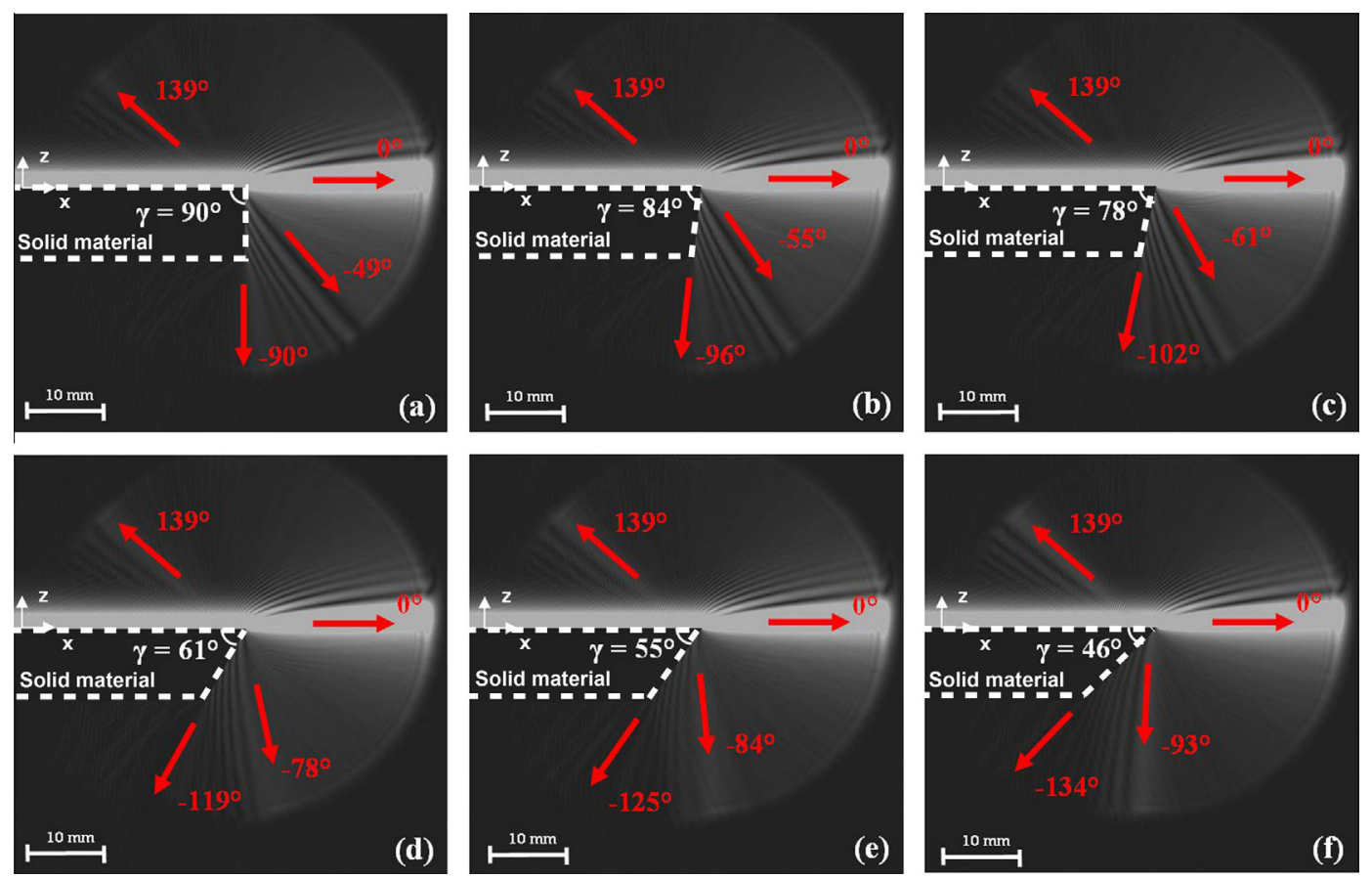

Fig. 11. Diffraction angles for a $5 \mathrm{MHz}$ Scholte-Stoneley wave in a brass-water system.

an upward directed trailing intensity lobe at $\theta=121^{\circ}$ in Fig. 10a, caused by the reflected Rayleigh wave, becomes stronger as $\gamma$ decreases.

\subsection{Material dependence of the reported phenomena}

The discussed phenomena remain clearly visible when different material combinations are investigated for decreasing $\gamma$ angles. The incorporation of the null-zone in the trailing field caused by the transmitted Rayleigh wave on the one hand and the intensity increase of the reflected Rayleigh wave on the other hand are also found when the material of the solid plate is changed. This is seen in Fig. 11, where the aluminum solid material is replaced by brass materials, while maintaining the $5 \mathrm{MHz}$ excitation frequency. The critical angles are obviously different from the ones discussed above as retrieved in each case separately. However, also here it is found that around a $\gamma$ angle of $72^{\circ}-\theta_{R}$, the same phenomena as those found above, are retrieved. 

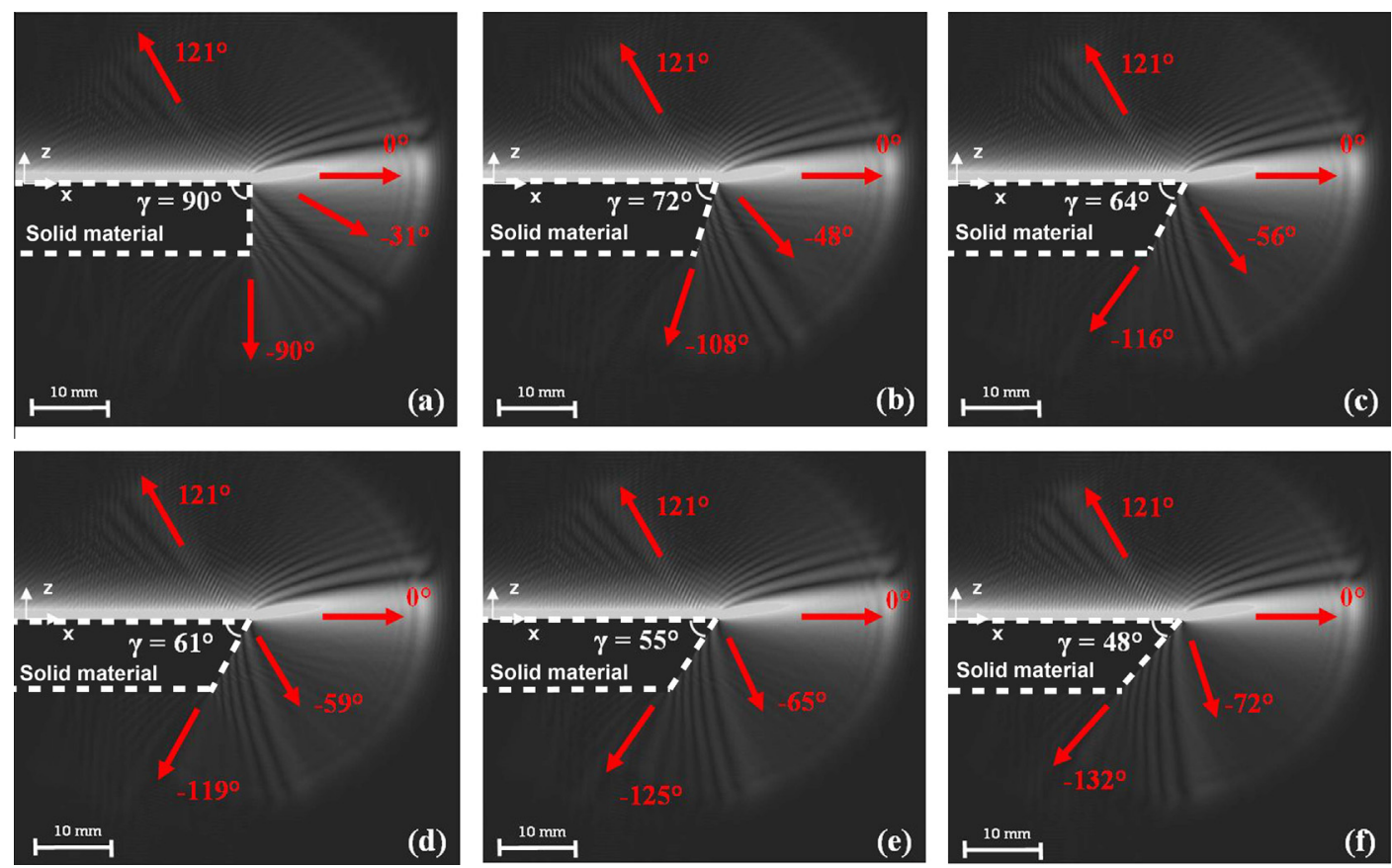

Fig. 12. Diffraction angles for a $3 \mathrm{MHz}$ Scholte-Stoneley wave in a aluminum-water system.

\subsection{Frequency dependence of the reported phenomena}

As a final verification of the discussed phenomena, the previous frequency of $5 \mathrm{MHz}$ is changed to $3 \mathrm{MHz}$ for the earlier aluminumwater system. In Fig. 12, apart from the obvious wavelength change of the incoming Scholte-Stoneley, similar diffraction phenomena as those found above, are also retrieved here.

\section{Conclusion}

The applied numerical method for the examination of acousticstructural problems allowed a thorough investigation of the interaction of Scholte-Stoneley waves with the corner and edge of an immersed dihedral solid. First the analytical equations for Scholte-Stoneley waves have been derived from the continuity conditions at the solid-liquid interface. The displacement equations have then been used as a boundary condition for Scholte-Stoneley wave generation along the interface. Consequently the numerically generated waves interacted with the corner of the dihedral and comparison with experimental results and earlier theoretical reports showed perfect agreement for a $90^{\circ}$ solid edge. In addition the study of the diffraction with dihedral angles $\gamma$ different from $90^{\circ}$ has been performed showing equally good results in comparison with experiments. Furthermore the existence of a critical dihedral angle was discovered, forming a transition between two diffraction phenomena.

\section{Acknowledgments}

The authors gratefully acknowledge financial support from the Fund for Scientific Research - Flanders (FWO) (Belgium) and also from the Georgia Tech Lorraine and le Conseil Régional de Lorraine (France).

\section{References}

[1] K. Gipson, P.L. Marston, Backscattering enhancements due to retroreflection of ultrasonic leaky rayleigh waves at corners of solid elastic cubes in water, J. Acoust. Soc. Am. 105 (2) (1999) 700-710.

[2] J.G. Scholte, On the Stoneley-wave equation i, Proc. K. Ned. Akad. Wetensc. 45 (1/5) (1942) 20-25.

[3] J.G. Scholte, On the Stoneley wave equation ii, Proc. K. Ned. Akad. Wetensc. 45 (1/5) (1942) 159-164.

[4] N. Declercq, R. Briers, J. Degrieck, O. Leroy, The history and properties of ultrasonic inhomogeneous waves, IEEE Trans. Ultrason. Ferroelectr. Freq. Control 52 (5) (2005) 776-791.

[5] L. Rayleigh, On the dynamical theory of gratings, Proc. Roy. Soc. Lond. Ser. Contain. Papers Math. Phys. Char. 79 (1907) 399-416.

[6] Lippmann, Note on the theory of gratings, J. Opt. Soc. Am. 43 (5) (1965) 408.

[7] J. Lekner, Theory of Reflection of Electromagnetic and Particle Waves, Martinus Nyhoff Publishers, Dordrecht, 1987.

[8] N.F. Declercq, J. Degrieck, R. Briers, O. Leroy, Theory of the backward beam displacement on periodically corrugated surfaces and its relation to leaky Scholte-Stoneley waves, J. Appl. Phys. 96 (11) (2004) 6869-6877.

[9] A. Tinel, J. Duclos, Diffraction and conversion of the Scholte-Stoneley wave at the extremity of a solid, J. Acoust. Soc. Am. 95 (1) (1994) 13-20.

[10] S. Nasr, J. Duclos, M. Leduc, PVDF transducers generating Scholte waves, Electron. Lett. 24 (6) (1988) 309-311.

[11] S.N. Guzhev, V.M. Levin, Generation of Stoneley surface acoustic-waves by an electrode transducer, Sov. Phys. Acoust.-Ussr 33 (4) (1987) 355-361.

[12] N.F. Declercq, A. Teklu, M.A. Breazeale, R. Briers, O. Leroy, J. Degrieck, et al., Study of the scattering of leaky rayleigh waves at the extremity of a fluidloaded thick plate, J. Appl. Phys. 96 (10) (2004) 5836-5840.

[13] R. Briers, O. Leroy, G.N. Shkerdin, Conversion of a Stoneley wave at the extremity of a fluid loaded plate, J. Acoust. Soc. Am. 101 (3) (1997) 1347-1357.

[14] N.F. Declercq, R. Briers, O. Leroy, J. Degrieck, G.N. Shkerdin, The radiation mode theory in ultrasonics, IEEE Trans. Ultrason. Ferroelectr. Freq. Control 52 (5) (2005) 802-808.

[15] E. Lamkanfi, N.F. Declercq, W. Van Paepegem, J. Degrieck, Finite element analysis of transmission of leaky Rayleigh waves at the extremity of a fluidloaded thick plate, J. Appl. Phys. 101 (11) (2007) 10. 